\title{
Thermal Vibration Amplitudes and Structure of As on Si(001)
}

\section{Citation}

Franklin, G. E., E. Fontes, Y. Qian, M. J. Bedzyk, J. A. Golovchenko, and J. R. Patel. 1994. Thermal Vibration Amplitudes and Structure of As on Si(001). Physical Review B 50, no. 11: 7483-7487. doi:10.1103/physrevb.50.7483.

\section{Published Version}

doi:10.1103/PhysRevB.50.7483

\section{Permanent link}

http://nrs.harvard.edu/urn-3:HUL.InstRepos:29407026

\section{Terms of Use}

This article was downloaded from Harvard University's DASH repository, and is made available under the terms and conditions applicable to Other Posted Material, as set forth at http:// nrs.harvard.edu/urn-3:HUL.InstRepos:dash.current.terms-of-use\#LAA

\section{Share Your Story}

The Harvard community has made this article openly available.

Please share how this access benefits you. Submit a story.

\section{Accessibility}




\title{
Thermal vibration amplitudes and structure of $\mathrm{As}$ on $\mathrm{Si}(001)$
}

\author{
G. E. Franklin \\ Department of Physics, Harvard University, Cambridge, Massachusetts 02138
}

E. Fontes

Cornell High-Energy Synchrotron Source, Ithaca, New York 14853

Y. Qian and M. J. Bedzyk

Department of Materials Science and Engineering, Northwestern University, Evanston, Illinois 60208 and Materials Science Division, Argonne National Laboratory, Argonne, Illinois 60439

J. A. Golovchenko

Department of Physics, Harvard University, Cambridge, Massachusetts 02138 and Rowland Institute for Science, Cambridge, Massachusetts 02142

\section{J. R. Patel*}

Sektion Physik der Universitat Munchen, Geschwister-Scholl-Platz 1, Munchen 80539, Germany

(Received 17 January 1994; revised manuscript received 14 April 1994)

Using the $\mathrm{x}$-ray standing-wave method, we have measured directly the thermal vibration amplitudes $\left\langle u^{2}\right\rangle$ of symmetric As dimers on a $\mathrm{Si}(001)-(2 \times 1)$ surface. For sample temperatures in the range $300 \mathrm{~K}$ $\leq T \leq 650 \mathrm{~K}$, the results are Debye-like. Above $650 \mathrm{~K}\left\langle u^{2}\right\rangle$ varies more rapidly, indicating the onset of defect-mediated processes. We also found that at room temperature the bond length of the As dimers is $2.58 \pm 0.04 \AA$ and that, independent of temperature, they sit $1.40 \pm 0.01 \AA$ above the top bulkextrapolated silicon (004) plane. These results provide a critical test for theoretical structure calculations.

In measuring the thermal vibration amplitudes of $\mathrm{Si}$ atoms at the $\mathrm{Si}(001)$ surface, ${ }^{1,2}$ one is confronted by the fact that, in addition to the vibration amplitude of individual atoms, there is superimposed a high-frequency (of order $10^{10} \mathrm{~Hz}$ ) switching motion ${ }^{3-8}$ of the reconstructed dimer pair at the surface. This switching motion occurs because the energetics of the buckled dimer can be described by a symmetric double-well potential where one of the dimer atoms is either in an up or a down state. In addition to the dimer motion, the average dimer separation is not fixed but seems to change with temperature. ${ }^{4}$

One means for simplifying the $\mathrm{Si}$ thermal vibration measurement is to modify the surface with an impurity. Such measurements have been performed with various adsorbates on $\mathrm{Si}(111)$ (Refs. 9 and 10) and show a change in the slope of the mean-square vibrational amplitude $\left\langle u^{2}\right\rangle$ versus temperature $T$ in the vicinity of $600 \mathrm{~K}$. For adsorbates on the $\mathrm{Si}(001)$ surface, though, where dimerization is the dominant mechanism for reducing the number of dangling bonds, $\left\langle u^{2}\right\rangle$ can easily be determined only if the impurity produces stable dimers (preferably symmetric) at the surface. One such possible isomorphous impurity is Ge. However, as we have shown earlier ${ }^{5}$ in conjunction with another $\mathrm{Si}(001)$ scanning tunneling microscopy (STM) result, ${ }^{4}$ this impurity produces asymmetric dimers that have a large displacement and are in a rapid switching motion at room temperature. Arsenic, on the other hand, generates dimers at the $\mathrm{Si}(001)$ surface that are similar to $\mathrm{Si}$ or $\mathrm{Ge}$ dimers but have the added virtue of being stable at room temperature. $^{11-13}$ In this study we report direct thermalvibration-amplitude measurements of $\mathrm{As}$ on $\mathrm{Si}(001)$ using the $\mathrm{x}$-ray standing-wave (XSW) method which is atom specific and records only signals from the As atoms. These measurements, in the temperature range 300 $\mathrm{K}<T<1000 \mathrm{~K}$, are of interest not only as a fundamental study of thermal motion at surfaces but also because of the insight they provide into defect-mediated processes. Our high-resolution measurements also determine the bond length and location of the As dimer relative to the Si lattice.

The X-ray standing-wave experiments were performed at the X15A beamline at the National Synchrotron Light Source (NSLS) at Brookhaven National Laboratory. Polished and Shiraki-etched $\mathrm{Si}(001)$ samples were inserted into our UHV system, with a base pressure of $5 \times 10^{-11}$ Torr. The samples were flashed at $925^{\circ} \mathrm{C}$ to remove the thin protective oxide layer. After cooling, low-energy electron diffraction (LEED) examination showed a twodomain $(2 \times 1)$ pattern characteristic of a clean $\mathrm{Si}(001)$ surface. The sample was then heated to $600^{\circ} \mathrm{C}$ and As was deposited onto the silicon substrate with the As metal evaporator source at $250^{\circ} \mathrm{C}$. During the 5-min deposition, the vapor pressure of As in the chamber rose to $1 \times 10^{-6}$ Torr. The As effusion cell was then shut off and the sample cooled to a holding temperature of $500^{\circ} \mathrm{C}$ until the As was pumped away (generally about $2 \mathrm{~h}$ ). This prevented the residual As in the chamber from adsorbing 
onto a cold sample substrate. The LEED pattern showed the same sharp $(2 \times 1)$ two-domain half-order spots as on the clean surface but with a significantly reduced background. This method of preparation produces an As saturation coverage of one monolayer. ${ }^{14}$

The x-ray measurements were performed at an energy of $17.5 \mathrm{keV}$ and at different sample temperatures. In general, uncertainties in the precise value of the feedbackcontrolled temperature were less than $25^{\circ} \mathrm{C}$. Whenever a new sample was prepared, standing-wave measurements were immediately recorded for the (004) and (022) reflections.

Figure 1 shows the room-temperature-measured (004) and (022) reflectivities as open squares and the corresponding As $K \alpha$ normalized fluorescence yields as open circles. The solid lines are calculated from the dynamical diffraction theory and fitted to the data. The two independent fitting parameters for each reflection, $\boldsymbol{P}_{\mathbf{H}}$ and $F_{H}$, are called the coherent position and coherent fraction, respectively. These parameters are the amplitude $\left(F_{H}\right)$ and phase $\left(P_{H}\right)$ of the $H$ th Fourier coefficient for the fluorescence-selected atom distribution and are determined by

$$
F_{\mathbf{H}} e^{i 2 \pi P} \mathbf{H}=\frac{C}{N} \sum_{n=1}^{N} e^{i 2 \pi \mathbf{H} \cdot r_{n}} e^{-\left(2 \pi^{2}\left\langle u_{n}^{2}\right\rangle\right) / d_{H}^{2}},
$$

where $C$ is the ordered fraction, $N$ is the number of fluorescing atoms within the unit cell, $\mathbf{H}$ is the

$$
1 \mathrm{ML} \mathrm{As} / \mathrm{Si}(001)-(2 \times 1)
$$

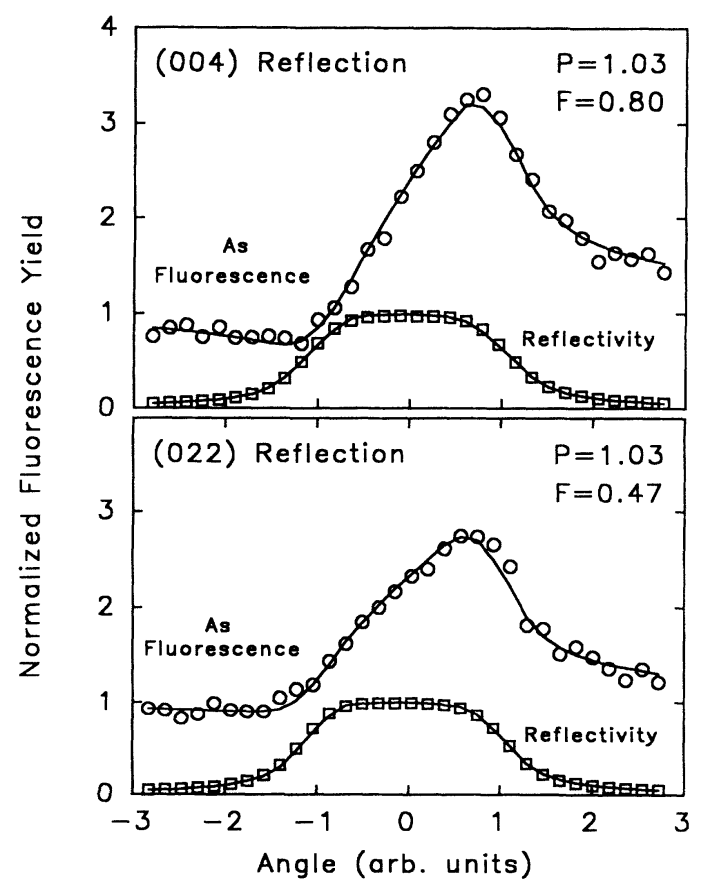

FIG. 1. The measured (004) and (022) reflectivity (open squares) and the corresponding As $K \alpha$ normalized fluorescence yields (open circles) for the saturation coverage of $\mathrm{As} / \mathrm{Si}(001)$ at room temperature. The solid lines are calculated from dynamical diffraction theory and fit to the data. The coherent position $(P)$ and coherent fraction $(F)$ have been determined from the fit. reciprocal-lattice vector for the $h k l$ diffraction planes whose $d$ spacing is $d_{H}, \mathbf{r}_{n}$ is the position of the $n$th atom, and $\left\langle u^{2}\right\rangle$ is the mean-squared vibration amplitude of the $n$th atom in the $\mathbf{H}$ direction.

From Figure 2 we can see the relevant geometry where the $\mathrm{Si}$ atoms (open circles) are presented in ideal bulklike positions and the As atoms (solid circles) are shown in a projected view. Thus, our measured coherent position of $P_{004}=1.03$ (Fig. 1) indicates that the position of the center of mass of the As atoms, relative to the (004) planes, is $3 \%(0.04 \AA)$ of a $d$ spacing above these bulkextrapolated planes $\left(d_{004}=1.36 \AA\right)$. Since there is a relaxation of the top Si layer, ${ }^{11-13}$ our $P$ values cannot be used to directly obtain the backbond distances between the $\mathrm{Si}$ and $\mathrm{As}$ atoms.

Using our four experimentally determined values $\left(P_{004}=1.03 \pm 0.01, F_{004}=0.80 \pm 0.01, P_{022}=1.03 \pm 0.01\right.$, and $F_{022}=0.47 \pm 0.02$ ), Eq. (1), and symmetry considerations of the dimer, we were able to uniquely determine by triangulation the As atom positions and dimer bond length. From an analysis of the data we find the dimer bond length $L=2.58 \pm 0.04 \AA$ for symmetric As dimers and these dimers sit $Z=1.40 \pm 0.01 \AA$ above the top (004) bulk-extrapolated plane.

Table I compares our results to a surface $\mathrm{x}$-ray diffraction measurement ${ }^{15}$ and to theoretically calculated model predictions for the As dimer bond length and the As dimer height above the bulk-extrapolated (004) silicon plane. For the height $Z$ of the As dimer, our result lies in closer agreement with the recent works of Kruger and Pollmann ${ }^{13}$ and Tang and Freeman. ${ }^{12}$ The theoretical approach of Ref. 13, while being similar in some details to Uhrberg et al. ${ }^{11}$ is sufficiently different to account for the calculated dimer height discrepancies in Table I.

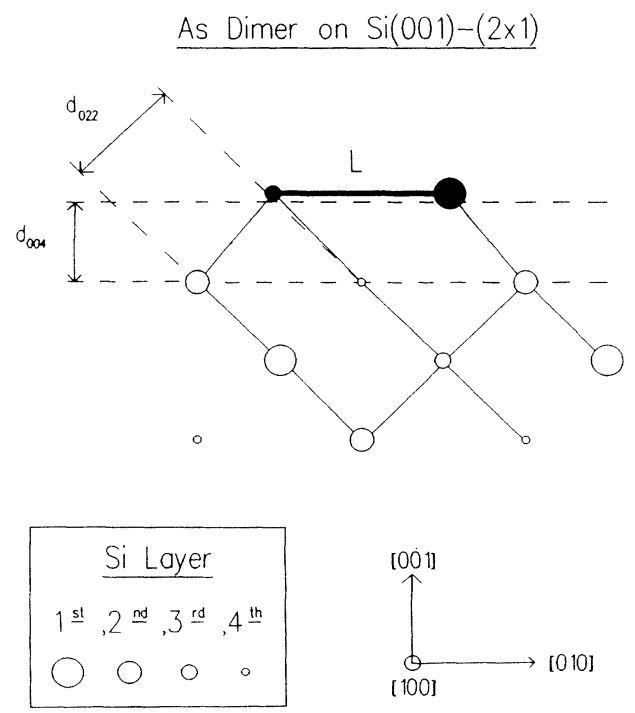

FIG. 2. Sideview of the projected As dimer (solid circles), with bond length $L$, sitting atop $\mathrm{Si}$ atoms (open circles) in ideal bulklike positions. The size of the circles are indicative of the different Si layers going into the page and away from the viewer. Shown are the relevant lattice spacings $d$ for the (004) and (022) reflections. 
TABLE I. Experimental and theoretical comparisons of the bond length $(L)$ and height $(Z)$ of the As dimer above the bulk-extrapolated Si(004) plane.

\begin{tabular}{lcc}
\hline \multicolumn{1}{c}{ Experiment and theory } & $L(\AA)$ & $Z$ ) \\
\hline Our results, XSW & $2.58 \pm 0.04$ & $1.40 \pm 0.01$ \\
Jedrecy (Ref. 15) XRD & $2.55 \pm 0.01$ & \\
Uhrberg et al. (Ref. 11) & 2.55 & 1.32 \\
Tang and Freeman (Ref. 12) & 2.52 & 1.37 \\
Kruger and Pollmann (Ref. 13) & 2.52 & 1.40 \\
\hline \hline
\end{tabular}

These differences mainly involve silicon atom relaxations under the As dimer and, in fact, if the downward relaxation of $0.09 \AA$ of the silicon atoms in Ref. 11 were ignored, agreement between theory and experiment would be exact. We trust that our position measurement for the As dimer will stimulate a critical examination of the different theoretical approaches and provide a standard for future comparisons with theory.

These results were also found to be independent of initial deposition temperatures in the range $500^{\circ} \mathrm{C}-700^{\circ} \mathrm{C}$. However, for growth temperatures around $400^{\circ} \mathrm{C}, F_{004}$ decreased to 0.57 (although $P_{004}$ remained the same at 1.03), indicating an increase in surface disorder. These conclusions are consistent with a previous STM study ${ }^{14}$ and are similar to an earlier standing-wave result ${ }^{16}$ $\left(P_{004}=1.04\right)$ of $\mathrm{As} / \mathrm{Si}(001)$ samples measured under ambient conditions.

In a general XSW analysis, the measured coherent fraction [from Eq. (1)] is given by

$$
F_{\mathrm{H}}=C a_{\mathrm{H}} D_{\mathrm{H}} \text {, }
$$

where $C$ (an ordered fraction), $a_{\mathbf{H}}$ (a geometrical factor), and $D_{H}$ (a Debye-Waller factor) are the three contributing factors. ${ }^{17}$ We have assumed that, for a given $\mathbf{H}$ direction, $\left\langle u^{2}\right\rangle$ is the same for each atom in the unit cell so that $D=\exp \left(-2 \pi^{2}\left\langle u^{2}\right\rangle / d^{2}\right)$. Since $a_{004}=1$ and $F_{004}=0.80$ for the As dimer, $C D_{004}=0.80$ [see Eq. (2)]. From a previous STM study ${ }^{14}$ it seems clear that the dimerized surface is highly ordered and therefore we assume $C=1$. We thus obtain a room-temperature rootmean-square thermal displacement $\sqrt{\left\langle u_{004}^{2}\right\rangle}$ of $0.14 \AA$. This is close to $0.12 \AA$, a value established for a variety of adsorbates on $\mathrm{Si}(111)$ surfaces. ${ }^{10}$ It is also close to values calculated for the $\mathrm{Si}$ dimer configuration on $\mathrm{Si}(001)$ and the $\mathrm{Si}(111)-(2 \times 1)$ surface. ${ }^{1,2}$ If we assume these smaller values for the thermal displacement, we can obtain limits of only a few percent on the possible amount of As disorder (i.e., $1-C$ ).

Assuming a Gaussian distribution for the thermal motion of the symmetric As dimer atoms, we have obtained $\left\langle u_{004}^{2}\right\rangle$ directly from the normalized $F_{004}$ values. A plot of the (004) (solid circles) and (022) (open circles) components of the mean-square vibration amplitude of the As atoms as a function of temperature in degrees Kelvin is shown in Fig. 3. The solid line through the points is a linear least-squares fit to the $\left\langle u_{004}^{2}\right\rangle$ versus $T$ data for $T<650 \mathrm{~K}$. For $T>650 \mathrm{~K}$ a second-order polynomial term was added though only to improve the goodness of the fit. The dashed line is a theoretical vibration- amplitude result from Mazur and Pollmann for the averaged (004) component (i.e., normal component) of the $\mathrm{Si}$ dimers on $\mathrm{Si}(001){ }^{2}$ The Alerhand, Joannopulos, and Mele $^{1}$ theoretical results (not shown) have a slope slightly less than that predicted by Ref. 2 .

It is apparent from Fig. 3 that the vibrational amplitude shows a different behavior above roughly $650 \mathrm{~K}$. From $300 \mathrm{~K}$ to $650 \mathrm{~K},\left\langle u_{004}^{2}\right\rangle$ versus $T$ is linear with a slope of

$$
(6.3 \pm 0.3) \times 10^{-5} \AA^{2} / K \text {, }
$$

which extrapolates to about zero at $T=0 \mathrm{~K}$ (neglecting the small zero-point oscillations of order $0.003 \AA^{2}$ from Mazur and Pollmann ${ }^{2}$ ). This follows the wellknown harmonic behavior predicted by Debye and we refer to the behavior in this regime as Debye-like. Theoretical results predict a linear behavior in this regime with a slope of $3.6 \times 10^{-5} \AA^{2} / \mathrm{K}^{2}$ Note that our results are for As adatoms and not Si adatoms. Above 650 $\mathrm{K},\left\langle u_{004}^{2}\right\rangle$ varies much more rapidly with temperature. We emphasize that the high-temperature behavior is not due to the onset of anharmonicity. If anharmonic effects were significant, we should observe a change in the coherent-position $\left(P_{004}\right)$ values. Within the experimental

Thermal Vibration Amplitudes for As on Si(001)

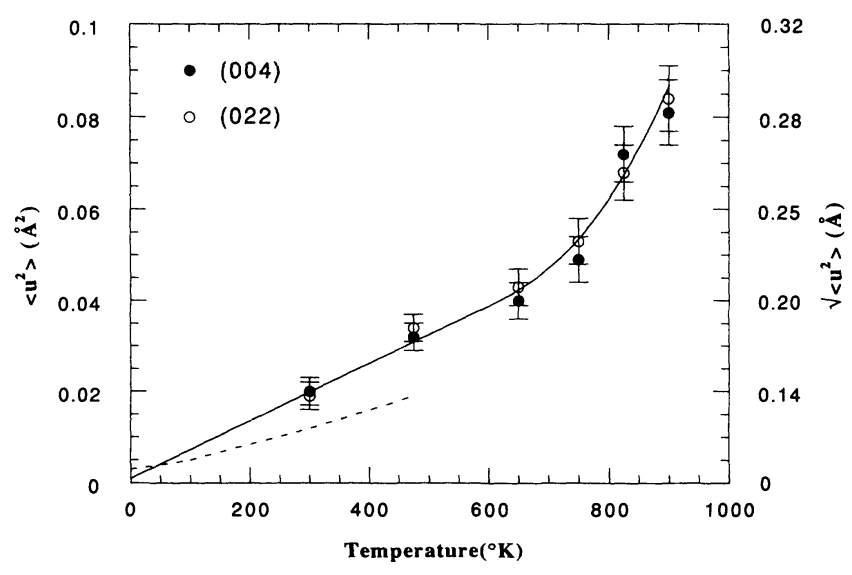

FIG. 3. The mean-square and root-mean-square vibration amplitudes as a function of temperature in degrees Kelvin. The $\left\langle u^{2}\right\rangle$ values from the (004) and (022) reflections are shown with their corresponding uncertainties as solid circles and open circles, respectively. The solid line is a least-squares fit to the (004) data and the dashed line is a theoretical result for $\mathrm{Si}$ dimers on Si(001)-(2×1) (Ref. 2). 
uncertainty, no such change was observed although the coherent fraction $\left(F_{004}\right)$ varied by a factor of 2 in the same temperature range. In addition, since the relative coherent position did not change as a function of temperature, the thermal coefficient of expansion of the surface structure in the [001] direction is not significantly different from that of bulk silicon.

Why then do we observe these two different regimes in $\left\langle u_{004}^{2}\right\rangle$ versus $T$ ? Theoretical calculations ${ }^{1,2}$ have been limited to the harmonic approximation and would predict a linear region extending beyond $650 \mathrm{~K}$. Recently, however, it has become increasingly evident that for $\mathrm{Si}$ at temperatures around $600 \mathrm{~K}$ there is an appreciable mobility of atoms at the surface. For instance, it is possible to grow good-quality epitaxial silicon at $600 \mathrm{~K}$ (Ref. 18) compared to the usual growth temperature around 1000 $\mathrm{K}$. We anticipate that the thermal vibration amplitudes at surfaces could be enhanced due to the presence of defects created by the mobility of Si atoms around $600 \mathrm{~K}$. Atoms in the vicinity of such defects could vibrate with large thermal vibration amplitudes, thus accounting for the enhanced $\left\langle u_{004}^{2}\right\rangle$. Other defect-related scenarios, which could account for the decreased coherent fraction at higher temperatures, involve either enhanced straininduced buckling of the As dimers or induced As disorder. In the latter scenario a simple calculation places the amount of As disorder around $20 \%$ for a temperature of $900 \mathrm{~K}$. Again, we point out that the defect production process does not change the average relative position $(P)$ values since these remain constant in the temperature range studied.

We now turn to the question of the anisotropy of the normal and in-plane measurements of the thermal vibration amplitudes on the Si(001) surface. For the Si(001)$(2 \times 1)$ surface, a convenient off-normal reciprocal-lattice vector is the (022), which is inclined at $45^{\circ}$ to the (001) surface. The projection of the normal component of the thermal vibration amplitude along (022) is substantial and, if the anisotropy is small, ${ }^{1,2}$ it will be difficult to separate the normal and in-plane components of $\left\langle u^{2}\right\rangle$. We find that the anisotropy [i.e., the (022) component in Fig. 3] is indeed rather small since the (004) and (022) values follow each other closely. Our results are in agreement with the theoretically calculated anisotropy for the $\mathrm{Si}(001)-(2 \times 1)$ surface which is about $6 \%$ at room tem- perature increasing to $10 \%$ at $450 \mathrm{~K} .^{2}$ This is in contrast to the $\mathrm{Si}(111)$ surface were we have observed a marked anisotropy for Ga and As absorbed species ${ }^{9,10}$ as a function of temperature.

It is not clear at present that, using the standing-wave technique and the instruments developed by us at NSLS, we can succeed in measuring such small differences. Perhaps higher-order reflections, with reciprocal-lattice vectors inclined at small angles to the surface, could in principle determine the small anisotropy exhibited on the $\mathrm{Si}(001)$ surface. In this case, however, the intensities will be substantially smaller than those for the lower-order Bragg reflections that we measured. It would appear that wiggler or undulator radiation from third-generation synchrotron sources such as European Synchrotron Radiation Facility, Advanced Photon Source, and Spring-8 would be ideal for such delicate measurements.

In conclusion, we have directly measured the meansquare thermal-vibration amplitude $\left\langle u^{2}\right\rangle$ as a function of temperature for the symmetric As dimers on a Si(001)$(2 \times 1)$ surface. From $300 \mathrm{~K}$ to $650 \mathrm{~K}$, the behavior of $\left\langle u^{2}\right\rangle$ was Debye-like with a slope greater than that predicted theoretically for Si dimers on the $\mathrm{Si}(001)$ surface. Above $650 \mathrm{~K}\left\langle u^{2}\right\rangle$ increased dramatically, suggesting the onset of enhanced surface-atom mobility and defectmediated processes. In addition, and as predicted by theory, the amount of anisotropy between the measured normal and in-plane $\left\langle u^{2}\right\rangle$ components was small. Our measured height of the As dimers above the bulkextrapolated $\mathrm{Si}(004)$ plane provides a critical test of theoretical structure calculations.

\section{ACKNOWLEDGMENTS}

We would like to acknowledge discussions with $\mathbf{J}$. Pollmann, J. Northrup, A. Mazur, and P. Kruger and we are indebted to Paul Freeland for the excellent sample preparations. This work was supported by the U.S. Department of Energy under Contract No. DEFG0289ER-45399 to Harvard University, under Contract No. W-31-109-ENG-38 to Argonne National Laboratory, and under Contract No. DE-AC02-76CH00016 to the facilities of the NSLS Brookhaven National Laboratory. M. J. Bedzyk received partial support from the NSF/MRL program at Northwestern University under Contract No. DMR-9120521.
*During the experiment the author was at AT\&T Bell Labs, Murray Hill, NJ 07974.

${ }^{1}$ O. L. Alerhand, J. D. Joannopulos, and E. J. Mele, Phys. Rev. B 39, 12622 (1989).

${ }^{2}$ A. Mazur and J. Pollmann, Surf. Sci. 225, 72 (1990).

${ }^{3}$ J. Dabrowski and M. Scheffler, Appl. Surf. Sci. 56-58, 15 (1992).

${ }^{4}$ R. A. Wolkow, Phys. Rev. Lett. 68, 2636 (1992).

${ }^{5}$ E. Fontes, J. R. Patel, and F. Comin, Phys. Rev. Lett. 70, 2790 (1993).

${ }^{6}$ F. H. Stillinger, Phys. Rev. B 46, 9590 (1992).

${ }^{7}$ G. P. Kochanski and J. E. Griffith, Surf. Sci. Lett. 249, L93
(1991).

${ }^{8}$ R. Rossmann, H. L. Meyerheim, V. Jahns, J. Wever, W. Moritz, D. Wolf, D. Dormisch, and H. Schulz, Surf. Sci. 279, 199 (1992).

${ }^{9}$ R. Martinez, E. Fontes, J. A. Golovchenko, and J. R. Patel, Phys. Rev. Lett. 69, 1061 (1992); R. Martinez and E. Fontes (private communications).

${ }^{10}$ E. Fontes, G. A. Gilmer, R. Martinez, J. A. Golovchenko, and J. R. Patel (unpublished).

${ }^{11}$ R. I. G. Uhrberg, R. D. Bringans, R. Z. Bachrach, and J. E. Northrup, Phys. Rev. Lett. 56, 250 (1986); J. Vac. Sci. Technol. A 4, 1259 (1986). 
12S. Tang and A. J. Freeman, Phys. Rev. B 48, 8068 (1993); S. Tang (private communication).

${ }^{13}$ P. Kruger and J. Pollmann, Phys. Rev. B 47, 1898 (1993); and (private communciations).

${ }^{14}$ R. S. Becker, T. Klitsner, and J. S. Vickers, J. Microsc. (Oxford) 152, 157 (1988).

${ }^{15}$ N. Jedrecy, M. Sauvage-Simkin, R. Pinchaux, J. Massies, N.
Greiser, and V. H. Etgens, Surf. Sci. 230, 197 (1990).

16J. Zegenhagen, J. R. Patel, B. M. Kincaid, J. A. Golovchenko, J. B. Mock, P. E. Freeland, and R. J. Malik, Appl. Phys. Lett. 53, 252 (1988).

${ }^{17}$ M. J. Bedzyk and G. Materlik, Phys. Rev. B 31, 4110 (1985).

${ }^{18}$ D. J. Eaglesham, H. J. Gossmann, and M. Cerullo, Phys. Rev. Lett. 65, 1127 (1990). 